University of Nebraska - Lincoln

DigitalCommons@University of Nebraska - Lincoln

August 1989

\title{
Thermochemistry of the gadolinium-copper interface
}

D. LaGraffe

Syracuse University

Peter A. Dowben

University of Nebraska-Lincoln, pdowben@unl.edu

M. Onellion

University of Wisconsin-Madison

Follow this and additional works at: https://digitalcommons.unl.edu/physicsdowben

Part of the Physics Commons

LaGraffe, D.; Dowben, Peter A.; and Onellion, M., "Thermochemistry of the gadolinium-copper interface" (1989). Peter Dowben Publications. 65.

https://digitalcommons.unl.edu/physicsdowben/65

This Article is brought to you for free and open access by the Research Papers in Physics and Astronomy at DigitalCommons@University of Nebraska - Lincoln. It has been accepted for inclusion in Peter Dowben Publications by an authorized administrator of DigitalCommons@University of Nebraska - Lincoln. 


\title{
Thermochemistry of the gadolinium-copper interface
}

\author{
D. LaGraffe and P. A. Dowben \\ Department of Physics, Syracuse University, Syracuse, New York 13244-1130 \\ M. Onellion \\ Department of Physics, University of Wisconsin, Madison, Wisconsin 53706
}

(Received 13 March 1989)

\begin{abstract}
We have characterized the thermochemistry of the gadolinium-copper interface using valenceband photoemission. We find that the copper substrate binds strongly with the gadolinium overlayer, based upon valence-band binding-energy shifts that occur with increasing gadolinium coverages on $\mathrm{Cu}(100)$. The net potential for copper at the interface is $3.82 \mathrm{eV} /$ atom which is some 0.51 $\mathrm{eV} /$ atom greater than the heat of sublimation for copper. The strong bonds formed between copper and gadolinium provide a thermochemical driving force for copper gadolinium-alloy formation. We observed a surface-to-bulk core-level shift for gadolinium of $0.3-0.4 \mathrm{eV}$. We also observed a $\mathrm{Cu}$ $3 d$ binding-energy increase of $0.73 \mathrm{eV}$ with increasing coverage.
\end{abstract}

The interaction of metal overlayers with metal substrates is a subject of increasing interest. ${ }^{1}$ For rare-earth overlayers, the chemical interaction of the rare-earth overlayer with the substrate is of importance because of the influence the substrate may exert upon the magnetic properties of the overlayer. ${ }^{2,3}$ For most transition metals, interdiffusion commonly occurs only at temperatures above $500 \mathrm{~K} .{ }^{4,5}$ For rare-earth overlayers, interdiffusion has been observed at significant rates at much lower temperatures. In the case of samarium overlayers on copper, copper has been reported to diffuse through the samarium overlayer at anomalously low temperatures. ${ }^{6}$

The binding-energy shifts of electronic states observed with increasing overlayer coverages have proved to be reliable indicators of surface-to-bulk core-level shifts ${ }^{7-14}$ as well as probes of the interface chemistry. ${ }^{12-16}$ If we assume complete electronic screening of the photohole, the binding-energy shifts can be used to describe the chemistry of the interface in terms of thermochemical quantities. ${ }^{73-15}$ The model used to determine the chemical shifts in the binding energies takes into account both the initial and final states of the photoemission process. ${ }^{17}$ This type of approach provides an accurate probe of the rare-earth-overlayer-substrate chemistry. The studies by other investigators of $\mathrm{Yb}$ on nickel ${ }^{14}$ and on silver ${ }^{12}$ have shown that the characterization of rare-earth core levels to be a profitable probe of the interface chemistry.

The angle resolved photoemission studies were performed at the Wisconsin $1 \mathrm{GeV}$ synchrotron in a chamber equipped with facilities for sample preparation, Auger-electron spectroscopy, and deposition capabilities in addition to photoemission and low-energy electron diffraction (LEED), as described previously. ${ }^{18}$ The hemispherical, angle resolved, electron energy analyzer was mounted on a double-axis goniometer and possessed a $3^{\circ}$ full-acceptance angle. The combined photon and analyzer resolution varied between 0.18 and $0.4 \mathrm{eV}$. Photoemission experiments were performed on the synchrotron $3-\mathrm{m}$ toroidal-grating monochromator. The angle- resolved, Auger-electron spectroscopy studies were undertaken with a hemispherical analyzer (Leybold EA10) in a separate vacuum chamber.

The gadolinium films were deposited as previously described $^{18}$ at a deposition rate of $1 \AA / \mathrm{min}$ onto samples cooled to between 150 and $200 \mathrm{~K}$ to preclude interdiffusion, though no difference in the electronic binding energies was observed with deposition at $150 \mathrm{~K}$ and deposition at $300 \mathrm{~K}$. The deposition cells were resistively heated tungsten filaments, precleaned by heating beyond the evaporant melting point and containing 99.9\%-pure gadolinium. We found it essential to cool every part of the deposition cell and enclosing shroud to obtain clean and reproducible depositions. Following considerable out-gassing of the source, clean depositions (as determined by Auger-electron spectroscopy) were observed, with the base pressure rising less that $1 \times 10^{-10}$ Torr during the evaporations.

In order to show that the diffusion of copper through gadolinium also occurs at anomalously low temperatures, as has been reported for samarium overlayers, ${ }^{6}$ angleresolved Auger-electron spectra were taken of gadolinium overlayers on $\mathrm{Cu}(100)$ at 20,60 , and $100^{\circ} \mathrm{C}$ as illustrated in Fig. 1. The ratio of the gadolinium 895-eV Auger-electron signal to the copper 920-eV Augerelectron signal, including corrections for sensitivity, increases with increasing emission angle. Ideally, with no interdiffusion, the gadolinium-to-copper signal should obey the following relationship: ${ }^{19}$

$$
I(x)=a+b /[\cos (x)]
$$

where $x$ is the emission angle from the normal.

Using a least-squares fit to the data obtained at 20 and $60^{\circ} \mathrm{C}$, a theoretical curve based upon the expected results with no interdiffusion has been generated for comparison with the data. These results indicate that copper interdiffusion with gadolinium overlayers occurs at substrate temperatures between 60 and $100^{\circ} \mathrm{C}$. Angle- 


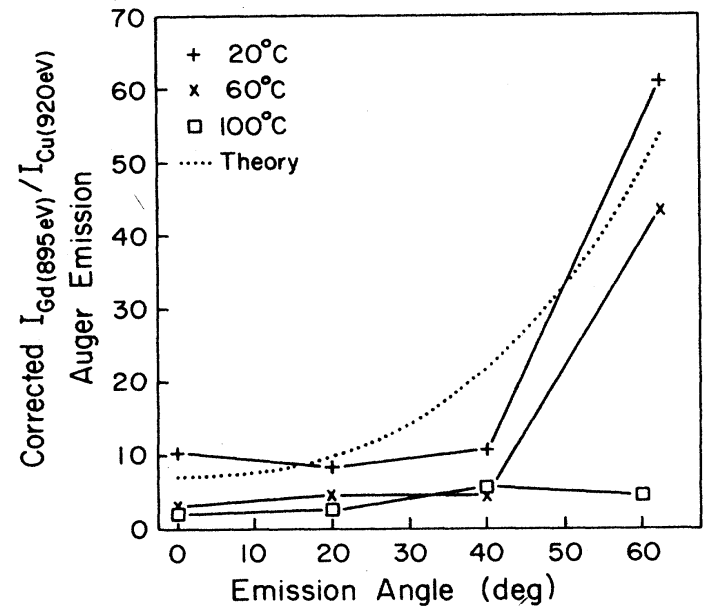

FIG. 1. Emission-angle dependence of the gadolinium 895$\mathrm{eV}$ Auger-electron signal with respect to the $\mathrm{Cu} 920-\mathrm{eV}$ Augerelectron signal. The results are plotted for several different annealing temperatures as indicated:,$+ 20^{\circ} \mathrm{C} ; \times, 60^{\circ} \mathrm{C} ; \square$, $100^{\circ} \mathrm{C}$. The results for 20 and $60^{\circ} \mathrm{C}$ are compared with a $1 / \cos (x)$ dependence as described in the text.

resolved, Auger-electron spectra of depositions onto a $\mathrm{Cu}(100)$ substrate at temperatures between 150 and 270 $\mathrm{K}$ indicate an abrupt $\mathrm{Gd}-\mathrm{Cu}$ interface.

Figure 2 illustrates the photoemission electron-energy distribution curves taken at normal emission for three different gadolinium overlayers of increasing thickness. The spectra exhibit features corresponding to the Gd $5 d$ electrons (0.3-0.4 eV binding energy), the $\mathrm{Cu} 3 d$ bands $(3.6 \mathrm{eV})$, and the $\mathrm{Gd} 4 f$ electrons $(8.6 \mathrm{eV})$. As an aside, the feature visible in some of the spectra at approximately $5.6-\mathrm{eV}$ binding energy is intrinsic, not due to contamination as discussed in detail elsewhere. ${ }^{18}$

The binding energies of both the $\mathrm{Cu} 3 d$ and the $\mathrm{Gd} 4 f$ levels shift with increasing gadolinium coverage. The $\mathrm{Cu}$ $3 d$ band for clean copper is about $3.0 \mathrm{eV}$ below the Fermi energy, while for 1-2-monolayer-thick gadolinium overlayers the $\mathrm{Cu} 3 d$ band binding energy is shifted to $3.73 \pm 0.05 \mathrm{eV}$. There is no significant $\mathrm{Cu} 3 d$ bindingenergy shift for gadolinium coverages greater than 1 monolayer. The Gd $4 f$ level shifts to smaller binding energies with increasing gadolinium coverages with a total shift of $0.37 \pm 0.09 \mathrm{eV}$. The Gd $4 f$-level binding-energy shift from 1 monolayer of gadolinium to 10 monolayers is $0.25 \pm 0.05 \mathrm{eV}$ and is less than the total binding-energy shift, since there is a measurable binding-energy shift (of more than $0.12 \pm 0.09 \mathrm{eV}$ ) associated with increasing coverages of $\mathrm{Gd}$ up to 1 monolayer. Figure 3 illustrates these changes in binding energy.

The binding-energy shifts for the $\mathrm{Gd} 4 f$ and $\mathrm{Cu} 3 d$ bands can be correlated with the gadolinium-overlayer thickness. Since we know from the studies summarized in Fig. 1 that interdiffusion does not occur under our deposition conditions, the results are not a consequence of intermetallic bulk compound formation.

The shift in the Gd $4 f$ level may be attributed to a combination of several effects. A surface-to-bulk corelevel shift of $0.48 \mathrm{eV}$ has been observed for metallic gadol-

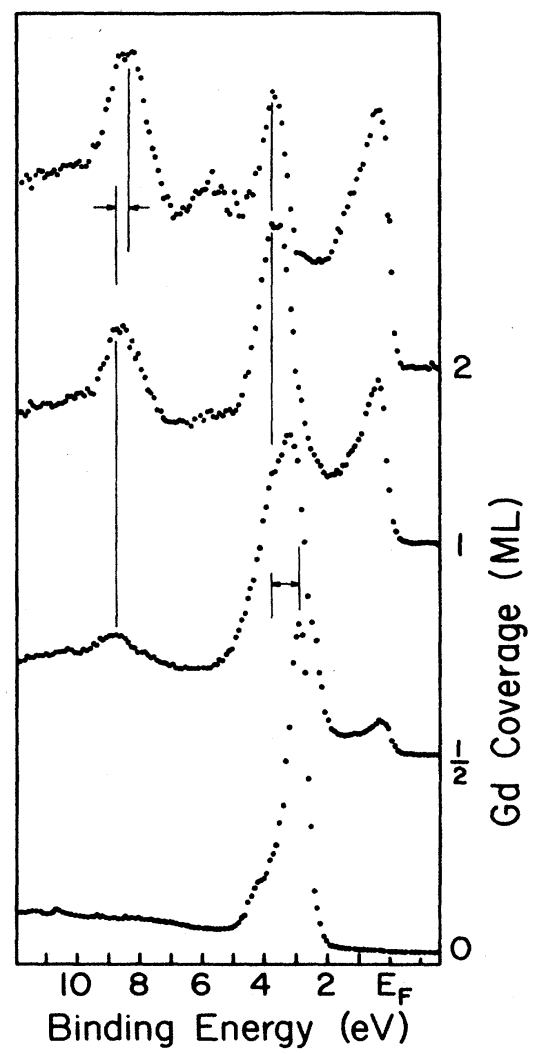

FIG. 2. Photoemission spectra of gadolinium overlayers on $\mathrm{Cu}(100)$ deposited at $150 \mathrm{~K}$. All spectra were taken at $h v=33$ $\mathrm{eV}$, with the photoelectrons collected normal to the surface. The clean $\mathrm{Cu}(100)$ surface is shown as is the surface following the deposition of $\frac{1}{2}, 1$, and 2 monolayers of gadolinium.

inium. ${ }^{19}$ Such a surface-to-bulk core-level shift would be observed with increasing gadolinium coverage as more photoemission-intensity contributions are contributed from "bulklike" gadolinium. Surface-to-bulk core-level shifts with increasing coverage are well documented for gold overlayers on platinum. 911 By subtracting the Gd $4 f$ spectrum of a 1-monolayer gadolinium overlayer from the spectrum of a 10-monolayer film, a surface-to-bulk core-level shift can be directly abstracted from the difference in the "bulk" and "surface" contributions. 13 Such a procedure has been undertaken as outlined in Fig. 4. This surface-to-bulk core-level shift of $0.3 \pm 0.07 \mathrm{eV}$ is consistent with our measured binding-energy shift of 0.25 $\mathrm{eV}$, but is smaller than the $0.48-\mathrm{eV}$ core-level shifts obtained from thicker films using deconvolution procedures. ${ }^{20}$ This indicates that gadolinium interactions of the copper substrate with the gadolinium overlayer cannot be ignored.

The small Gd $4 f$-level binding-energy shift of $0.1-0.2$ $\mathrm{eV}$ from dilute gadolinium coverages to 1 monolayer can be attributed to the effects of edges as has been done for submonolayer coverages of gold on platinum ${ }^{9,11}$ or as a result of dipole interactions. Halogen overlayers on a variety of substrates have also been observed to have decreasing core-level binding energies with increasing coverage (Ref. 21 and references therein). Previous au- 


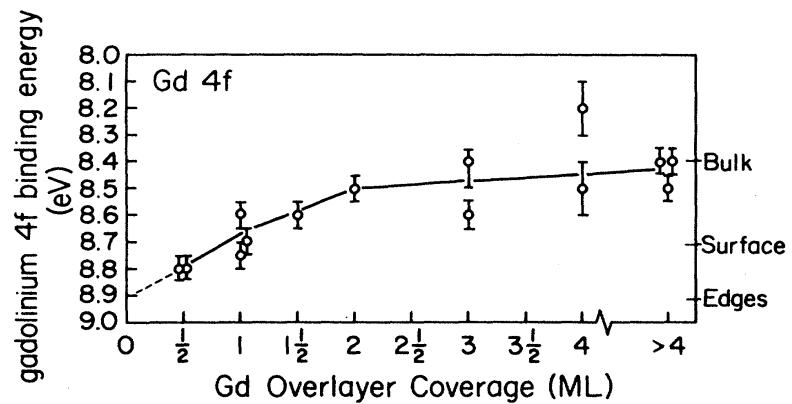

calculated. Note that neglecting polarization is sometimes a serious approximation ${ }^{25}$ and the result can differ substantially from the expected values. ${ }^{21}$

The calculation based upon dipole-dipole interactions alone predicts a charge transfer from the substrate to the gadolinium overlayer. This is in contrast to what one would expect when considering the work-function difference between gadolinium and copper. Gadolinium has a work function between 2.9 and $3.1 \mathrm{eV}$ (Refs. 26 and 27) and the $\mathrm{Cu}(100)$ substrate work function is $4.59 \mathrm{eV} .^{28}$ With this substantial difference in work functions, one would predict a charge transfer from the gadolinium overlayer to the substrate, causing an increase in the binding energies of the gadolinium states particularly at low coverages. The dipole-dipole calculations are invalidated if there are pronounced interfacial hybridization effects or if the surface reconstructs due to surface compound formation. The results of the angle-resolved Auger-electron-spectroscopy studies rule out compound formation as the source of this disparity. The slightly smaller than expected surface-to-bulk core-level shift as well as the anomalous shifts at submonolayer coverages indicate strong interactions of the gadolinium overlayer with the copper substrate. We conclude that the binding-energy shifts cannot be explained by a simple charge-transfer picture and are a result of more complicated gadolinium copper hybridizations.

Strong copper interactions with the gadolinium overlayer are indicated by the very large $\mathrm{Cu} 3 d$ bindingenergy shifts. The surface-to-bulk core-level shift for copper is about $0.22 \mathrm{eV}^{29,30}$ Such a core-level shift would be observed with increasing gadolinium coverage up to about a monolayer, ${ }^{7,13}$ yet this can only account for $0.22 \mathrm{eV}$ of the $0.73-\mathrm{eV}$ shift observed. The remaining $\sim 0.5 \mathrm{~V}$ binding-energy shift must be a result of the solid-state heat interaction between copper and gadolinium following the prescription of Egelhoff. ${ }^{13}$ If the heat of interaction of copper with copper is approximately the heat of sublimation [3.29 eV/atom (Ref. 31)], then the interaction of copper with gadolinium of about 3.8 $\mathrm{eV} /$ atom $(3.29+0.51 \mathrm{eV})$ is close to the interaction of gadolinium with gadolinium [4.15 eV/atom (Ref. 31)]. Interfacial hybridization substantially lowers the energy barriers to $\mathrm{Cu}-\mathrm{Cu}$ bond cleavage when $\mathrm{Gd}-\mathrm{Cu}$ bond formation is the alternative. Note that the strong contribution to the photoemission intensity of the $\mathrm{Cu} 3 d$ band for surface-shifted components of clean $\mathrm{Cu}(100)$ would act to reduce the observed surface-to-bulk shift, resulting, then, in an even larger solid-state heat of interaction.

This thermochemical driving force acts to lower the heat of activation for diffusion of copper through gadolinium. Since there is little doubt that there is rapid diffusion of copper through gadolinium at $100^{\circ} \mathrm{C}$ and through samarium at room temperature ${ }^{6}$ we postulate that the driving force for diffusion is the very large solidstate heat of interaction at the interface.

In conclusion, a solid-state heat of interaction between copper and gadolinium is $0.51 \mathrm{eV}$ or more. Copper diffusion through gadolinium occurs readily at $100^{\circ} \mathrm{C}$ and is driven by the very large heat of interaction. The Gd $4 f$ binding-energy shift is more than $0.37 \pm 0.09 \mathrm{eV}$ with in- 
creasing coverage on $\mathrm{Cu}(100)$. For gadolinium on $\mathrm{Cu}(100), 0.3 \mathrm{eV}$ of the Gd $4 f$ binding-energy shift may be attributable to a shift akin to the surface-to-bulk corelevel shift, while $0.1-0.2 \mathrm{eV}$ of the shift occurs at submonolayer coverages. Given that rare-earth metals such as $\mathrm{Gd}, \mathrm{Yb}$, and $\mathrm{Sm}$ readily interdiffuse with a wide variety of substrates, studies of the electronic structure of rare-earth overlayers must be undertaken with caution. Further complications in investigating rare-earth overlayers are indicated by recent studies ${ }^{18}$ showing that gadolinium overlayers hybridize differently with copper and nickel substrates.

The authors would like to thank Y. J. Kime for her assistance with the dipole-dipole calculations and D. M. Wieliczka and C. G. Olson for providing the results of their work prior to publication. This work was funded by the U.S. Department of Energy. We would like to thank the staff of the Synchrotron Radiation Center, Stoughton, WI, where this work was carried out. The Synchrotron Radiation Center is funded by the National Science Foundation (NSF).
${ }^{1}$ For a recent review and extensive references, see $\mathbf{P}$. A. Dowben, M. Onellion, and Y. J. Kime, Scanning Microsc. 2, 177 (1988).

2P. A. Dowben, Shikha Varma, Y. J. Kime, D. R. Mueller, and M. Onellion, Z. Phys. B 73, 247 (1988).

${ }^{3}$ C. Carbone and E. Kisker, Phys. Rev. B 36, 1280 (1987).

${ }^{4}$ P. A. Dowben, A. Miller, and R. W. Vook, Gold Bull. 20, 54 (1987); P. A. Dowben, D. Wright, and M. Grunze, Surf. Sci. 134, L524 (1983).

5J. Mazaruski and P. A. Dowben, in Surface Segregation and Related Phenomenon, edited by P. A. Dowben and A. Miller (Chemical Rubber Co., Cleveland, in press).

${ }^{6}$ D. M. Wieliczka and C. G. Olson (unpublished).

${ }^{7}$ W. F. Egelhoff, Jr., Surf. Sci. Rep. 6, 253 (1987).

${ }^{8}$ Tai-chang Chiang, CRC Crit. Rev. Solid-State Mater. Sci. 14, 269 (1988).

${ }^{9}$ M. Salmeron, S. Ferrer, M. Jazzar, and G. A. Somorjai, Phys. Rev. B 28, 1158 (1983).

${ }^{10}$ S. B. DiCenzo, P. H. Citrin, E. H. Hartford, Jr., and G. K. Wertheim, Phys. Rev. B 34, 1343 (1986).

${ }^{11}$ M. Salmeron, S. Ferrer, M. Jazzar, and G. A. Somorjai, Phys. Rev. B 28, 6758 (1983).

${ }^{12}$ A. Nilsson, N. Martensson, J. Hedman, B. Eriksson, R. Bergman, and U. Gelius, Surf. Sci. 162, 51 (1985).

${ }^{13}$ W. F. Egelhoff, Jr., Phys. Rev. Lett. 50, 587 (1983).

${ }^{14}$ A. Nilsson, B. Eriksson, N. Martensson, J. N. Andersen, and J. Onsgaard, Phys. Rev. B 38, 10357 (1988); I. Chorkendorff, J. Onsgaard, J. Schmidt-May, and R. Nyholm Surf. Sci. 160, 587 (1985)

${ }^{15}$ J. C. Hansen, J. A. Benson, W. D. Clendening, M. T. McEllistrem, and J. G. Tobin, Phys. Rev. B 36, 6186 (1987).
${ }^{16}$ A. Fåldt and H. P. Myers, Phys. Rev. Lett. 52, 1315 (1984).

${ }^{17}$ B. Johansson and N. Martensson, Phys. Rev. B 21, 4427 (1980).

${ }^{18}$ P. A. Dowben, D. LaGraffe, and M. Onellion, J. Phys. Solid State (to be published).

${ }^{19}$ P. A. Dowben, Phys. Rev. B 30, 7278 (1984); C. S. Fadley, in Electron Spectroscopy: Theory, Techniques and Applications, edited by C. R. Brundle and A. D. Baker (Academic, New York, 1978), Vol. 2.

${ }^{20}$ R. Kammerer, J. Barth, F. Gerken, A. Flödstrom, and L. I. Johansson, Solid State Commun. 41, 435 (1982).

${ }^{21}$ P. A. Dowben, Y. J. Kime, D. Mueller, and T. N. Rhodin, J. Chem. Phys. 89, 4406 (1988).

${ }^{22}$ C. Somerton, C. F. McConville, D. P. Woodruff, and R. G. Jones, Surf. Sci. 136, 23 (1984).

${ }^{23}$ S. B. DiCenzo, G. K. Wertheim, and D. N. E. Buchanan, Surf. Sci. 121, 411 (1982)

${ }^{24}$ S. B. DiCenzo, G. K. Wertheim, and D. N. E. Buchanan, Phys. Rev. B 24, 6143 (1981).

${ }^{25}$ N. D. Lang and A. R. Williams, Phys. Rev. B 16, 2408 (1977).

${ }^{26}$ D. Eastman, Phys. Rev. B 2, 1 (1970).

${ }^{27}$ R. L. Ramey and S. J. Katzberg, J. Chem. Phys. 53, 1347 (1970).

${ }^{28}$ P. O. Gartland, S. Berge, and B. J. Slagsvold, Phys. Norv. 7, 39 (1973).

${ }^{29}$ W. F. Egelhoff, Phys. Rev. B 29, 4769 (1984).

${ }^{30}$ P. H. Citrin, G. K. Wertheim, and Y. Baer, Phys. Rev. B 27, 3160 (1983); P. H. Citrin and G. K. Wertheim, ibid. 27, 3176 (1983).

${ }^{31}$ Handbook of Chemistry and Physics (Chemical Rubber Co., Cleveland, 1988). 